\title{
Osteoporosis and strontium-substituted hydroxyapatites
}

\author{
Nikolaos Kourkoumelis \\ Department of Medical Physics, Faculty of Medicine, School of Health Sciences, University of Ioannina, Ioannina, Greece \\ Correspondence to: Nikolaos Kourkoumelis. Department of Medical Physics, Faculty of Medicine, School of Health Sciences, University of Ioannina, \\ 45110 Ioannina, Greece. Email: nkourkou@uoi.gr.
}

Submitted Jul 17, 2016. Accepted for publication Jul 25, 2016.

doi: 10.21037/atm.2016.10.03

View this article at: http://dx.doi.org/10.21037/atm.2016.10.03

Biomedical implants able to stimulate sufficient osteointegration in the vicinity of the bone-material interface are essential for reducing the patient's immobility time and for stabilizing the implant fixation in the long term. Hydroxyapatite (HA) is a biocompatible and osteoconductive material which exhibits high affinity for host bone tissues through mineral surface reactions. The mechanism of the formation and strengthening of the boneHA interface was studied using high-resolution transmission electron microscope (HRTEM) and energy-dispersive X-ray (EDX) analysis (1) and it was suggested that the transition of crystalline to amorphous HA is the first critical step in the bone-implant bonding.

Osteoporosis is typically manifested by the systemic impairment of bone mass and microarchitecture (2). Bone quality deterioration can severely compromise the supporting ability of an implant especially in the cancellous bone area (3). The reduced bone mass resulting from the imbalanced bone resorption in osteoporotic bones can lead to failure in the immediate peri-implant bone region and ultimately to fixation failure. Non-resorbable HA particles have shown promising results improving osteointegration with a denser peri-implant bone structure (4) while clinical tests showed that the HA-coated prosthesis is beneficial to total-hip arthroplasty (5). Even though HA based coatings improve bone formation around implants, osteoporotic bones prevent the stable mechanical fixation for reasons largely unknown at the microscale but probably due to the decreased bone volume and structural deterioration apparent at the macroscale (6).

Bone mineral comprises of small crystallites of carbonate apatite, which is essentially calcium phosphate HA $\left[\mathrm{Ca}_{10}\left(\mathrm{PO}_{4}\right)_{6}(\mathrm{OH})_{2}\right]$, where carbonate groups have replaced a fraction of phosphates and/or hydroxyl groups in the structure. The structure of HA has a hexagonal close packed arrangement with $\mathrm{P} 63 / \mathrm{m}$ symmetry although monoclinic variants have also been proposed as energetically feasible. For pure HA the cell dimensions are $9.42 \AA$ for the a- and b-lattice spacing and $6.88 \AA$ for the c-lattice spacing (ICDD PDF-4 database, 00-055-0592). The lattice parameters of the apatite crystals largely depend on chemical substitutions for $\mathrm{Ca}^{2+}, \mathrm{OH}^{-}$and $\mathrm{PO}_{4}^{3-}$. The tendency for cationic and anionic substitutions is driven by the large ratio of surface area to volume which promotes the swift atom absorption and dissolution. The unit cell of pure HA has two different (i.e., non-equivalent) crystallographic sites for the ten $\mathrm{Ca}$ atoms: four 9-fold coordinated Ca1 polyhedra and six 7-fold coordinated $\mathrm{Ca} 2$ polyhedra (Figure 1) which form a channel along the c-crystallographic axis (7).

The osteointegration of an implant as a function of the biological response of HA can be significantly enhanced by pharmaceutical agents and/or doping HA with various ions (8). These substitutions can alter important physicochemical properties of the bone mineral like surface reactivity, thermal stability, and solubility rates (9). In particular, solubility is largely affected by porosity and crystalline properties (size and perfection). The latter vary inversely to chemical reactivity and it was found that apatites obtained by partial substitution of $\mathrm{Ca}^{2+}$ by $\mathrm{Sr}^{2+}$ yield higher solubility compared to pure HA without inducing any changes in the crystal structure (10). Both strontium and calcium belong to the group 2 of the periodic table and have common chemical properties. In this respect, strontium is characterized as "a bone seeking element" having similar charge/size ratio with calcium. Almost the total body content of strontium is in the skeleton although in small quantities compared to calcium. However, the amount of strontium in new bone is higher compared to old bone (11) and it is mainly distributed 


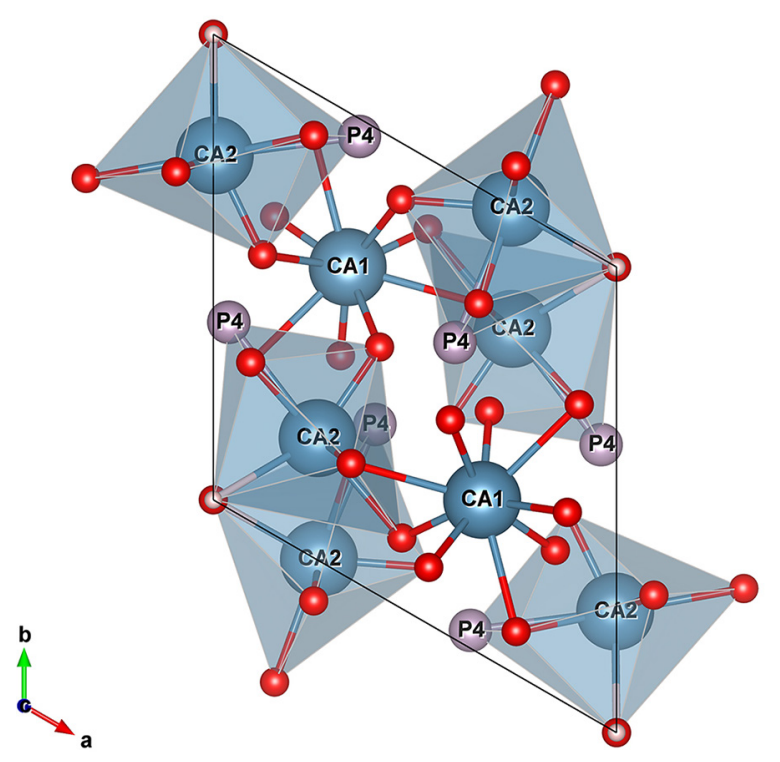

Figure 1 The unit cell of stoichiometric HA along the c-axis. HA, hydroxyapatite.

into trabecular sections (12). Strontium is suggested to be beneficial for bone in postmenopausal osteoporosis. $\mathrm{Sr}$ (II) salts have been shown to assist the replication of pre-osteoblastic cells promoting bone formation (13) and to a lesser extent, decrease bone resorption in vivo (14). In this way, a net gain in bone mineral density (BMD) during bone turnover is achieved, albeit in an uncoupled manner during the so-called "anabolic window" when bone formation autonomously surpasses bone resorption. Oral administration of $\mathrm{Sr}$-salts results in adsorption of $\mathrm{Sr}$ ions on the apatite surface or substitution of $\mathrm{Ca}$ ions in the apatite crystal lattice at low ion exchange rates. Approximately only one in ten $\mathrm{Ca}$ ions is substituted by $\mathrm{Sr}$ ions (15). The latter procedure of ion exchange is more profoundly realized in new bone during remodeling. The effects of strontium on bone are dose dependent and although low doses stimulate bone formation, high doses have deleterious effect on bone mineralization $(16,17)$. Prescription of Sr-salts acting as antiosteoporotic drugs is not widely acceptable and it is still under debate $(18,19)$. On the contrary, a lot of attention has been given to osteoporotic fracture repair by Sr modified bioceramics with improved osteointegration capabilities and minimal failure rates of implant fixation. Moreover, biocompatible Sr-doped HAs enhance the peri-implant bone formation more efficiently than administered strontium (20).

Li et al. (21) recently published a thought-provoking study to assess cancellous bone response to strontiumdoped hydroxyapatite (SrHA) in ovariectomized (OVX) rats. They randomized twenty 3-month-old female Sprague Dawley rats with bilateral ovariectomy into two equal groups depending on the type of implants they received in the right distal femur after the application of the osteoporotic protocol. The implants were HA and SrHA (HA with $10 \mathrm{~mol} \%$ calcium substituted by strontium) prepared by a chemical precipitation method and calcined to $1,050{ }^{\circ} \mathrm{C}$ for 0.5 -hour. The implants specimens were fully characterized by multiple techniques. The surface topology was evaluated by scanning electron microscopy (SEM), the structural characteristics by X-ray powder diffraction (XRD), and the elemental ratio of $\mathrm{Ca}$ and $\mathrm{Sr}$ by EDX and inductively coupled plasma mass spectrometry (ICP-MS). The bone-implant interface was assessed 8 weeks after the implantation by micro-CT and histological image analysis. Results showed that SrHA is superior to stoichiometric HA in every parameter studied. Thus, the percentage bone volume, the bone-to-implant contact and the bone area density were increased by $>40 \%$, the mean trabecular thickness and the mean connectivity density by $>30 \%$, while the mean trabecular separation decreased by $\sim 20 \%$. The exact reason why SrHA improves the peri-implant bone density as well as the cancellous microarchitecture is still not known. Between the two HA types, no significant differences were observed in surface morphology and no secondary phases were detected after the Sr doping. On the other hand, crystalline size was increased, as expected, due to the incorporation of strontium atoms having larger atomic radius. In this work the beneficial effect of SrHA was attributed to the chemical properties of the strontium itself. A number of parameters have been considered in the literature which may partially explain the positive response of Sr-doped HA implants. Crystallinity is closely related to the dissolution of HA and in vivo studies have shown that HA coating of low crystallinity promotes faster initial bone fixation but higher crystallinity is better for longterm implantation (22). Moreover, for carbonated apatites, microstrain rather than crystallite size, plays the major role in solubility (23). Although the mechanism for the enhanced osteointegration at the bone-implant interface in the presence of strontium is unclear, the degree of substitution seems to be critical. Recent studies (24) indicate that SrHAs with approximately molar ratio of $\mathrm{Sr} / \mathrm{Ca}=0.1$ are not cytotoxic, they do not alter the structural properties of the HA and effectively promote osteogenesis not only in shortterm osteoporotic rat models but also in long-term induced 
osteoporosis in an aged rat model. Finally, a new study (25) showed that $10 \%$ SrHA coating exhibited the best results for Ti-implants osteointegration and fixation compared with the analogous $\mathrm{Zn}$ and $\mathrm{Mg}$ HA-coatings in osteoporotic rats.

The article by Li et al. clearly shows that SrHA is a promising material for bone tissue engineering which promotes osteogenesis and improves the trabecular microarchitecture under osteoporotic conditions.

\section{Acknowledgements}

None.

\section{Footnote}

Provenance: This is a Guest Editorial commissioned by Section Editor Xianzuo Zhang, MD (Department of Orthopedics and Arthroplasy, Nanfang Hospital, Southern Medical University, Guangzhou, China).

Conflicts of Interest: The author has no conflicts of interest to declare.

Comment on: Li Y, Luo E, Zhu S, et al. Cancellous bone response to strontium-doped hydroxyapatite in osteoporotic rats. J Appl Biomater Funct Mater 2015;13:28-34.

\section{References}

1. Chen QZ, Wong CT, Lu WW, et al. Strengthening mechanisms of bone bonding to crystalline hydroxyapatite in vivo. Biomaterials 2004;25:4243-54.

2. Kourkoumelis N, Lani A, Tzaphlidou M. Infrared spectroscopic assessment of the inflammation-mediated osteoporosis (IMO) model applied to rabbit bone. J Biol Phys 2012;38:623-35.

3. Motohashi M, Shirota T, Tokugawa $\mathrm{Y}$, et al. Bone reactions around hydroxyapatite-coated implants in ovariectomized rats. Oral Surg Oral Med Oral Pathol Oral Radiol Endod 1999;87:145-52.

4. Tami AE, Leitner MM, Baucke MG, et al. Hydroxyapatite particles maintain peri-implant bone mantle during osseointegration in osteoporotic bone. Bone 2009;45:1117-24.

5. Tao ZS, Zhou WS, Tu KK, et al. The effects of combined human parathyroid hormone (1-34) and simvastatin treatment on osseous integration of hydroxyapatite-coated titanium implants in the femur of ovariectomized rats.
Injury 2015;46:2164-9.

6. Mueller TL, Basler SE, Müller R, et al. Time-lapsed imaging of implant fixation failure in human femoral heads. Med Eng Phys 2013;35:636-43.

7. Bigi A, Boanini E, Capuccini C, et al. Strontiumsubstituted hydroxyapatite nanocrystals. Inorg Chim Acta 2007;360:1009-16.

8. Evis Z, Webster TJ. Nanosize hydroxyapatite: doping with various ions. Advances in Applied Ceramics 2011;110:311-21.

9. Terra J, Dourado ER, Eon JG, et al. The structure of strontium-doped hydroxyapatite: an experimental and theoretical study. Phys Chem Chem Phys 2009;11:568-77.

10. Zhang W, Shen Y, Pan H, et al. Effects of strontium in modified biomaterials. Acta Biomater 2011;7:800-8.

11. Marie PJ, Ammann P, Boivin G, et al. Mechanisms of action and therapeutic potential of strontium in bone. Calcif Tissue Int 2001;69:121-9.

12. Boivin G, Deloffre P, Perrat B, et al. Strontium distribution and interactions with bone mineral in monkey iliac bone after strontium salt (S 12911) administration. J Bone Miner Res 1996;11:1302-11.

13. Canalis E, Hott M, Deloffre P, et al. The divalent strontium salt S12911 enhances bone cell replication and bone formation in vitro. Bone 1996;18:517-23.

14. Buehler J, Chappuis P, Saffar JL, et al. Strontium ranelate inhibits bone resorption while maintaining bone formation in alveolar bone in monkeys (Macaca fascicularis). Bone 2001;29:176-9.

15. Pors Nielsen $S$. The biological role of strontium. Bone 2004;35:583-8.

16. Dahl SG, Allain P, Marie PJ, et al. Incorporation and distribution of strontium in bone. Bone 2001;28:446-53.

17. Grynpas MD, Hamilton E, Cheung R, et al. Strontium increases vertebral bone volume in rats at a low dose that does not induce detectable mineralization defect. Bone 1996;18:253-9.

18. Cianferotti L, D'Asta F, Brandi ML. A review on strontium ranelate long-term antifracture efficacy in the treatment of postmenopausal osteoporosis. Ther Adv Musculoskelet Dis 2013;5:127-39.

19. Pernicova I, Middleton ET, Aye M. Rash, strontium ranelate and DRESS syndrome put into perspective. European Medicine Agency on the alert. Osteoporos Int 2008;19:1811-2.

20. Landi E, Tampieri A, Celotti G, et al. Sr-substituted hydroxyapatites for osteoporotic bone replacement. Acta Biomater 2007;3:961-9. 
21. Li Y, Luo E, Zhu S, et al. Cancellous bone response to strontium-doped hydroxyapatite in osteoporotic rats. J Appl Biomater Funct Mater 2015;13:28-34.

22. Xue W, Liu X, Zheng X, et al. Effect of hydroxyapatite coating crystallinity on dissolution and osseointegration in vivo. J Biomed Mater Res A 2005;74:553-61.

23. Baig AA, Fox JL, Young RA, et al. Relationships among carbonated apatite solubility, crystallite size, and

Cite this article as: Kourkoumelis N. Osteoporosis and strontium-substituted hydroxyapatites. Ann Transl Med 2016;4(Suppl 1):S10. doi: 10.21037/atm.2016.10.03 microstrain parameters. Calcif Tissue Int 1999;64:437-49.

24. Chandran S, Babu SS, Vs HK, et al. Osteogenic efficacy of strontium hydroxyapatite micro-granules in osteoporotic rat model. J Biomater Appl 2016. [Epub ahead of print].

25. Tao ZS, Zhou WS, He XW, et al. A comparative study of zinc, magnesium, strontium-incorporated hydroxyapatitecoated titanium implants for osseointegration of osteopenic rats. Mater Sci Eng C Mater Biol Appl 2016;62:226-32. 